\title{
Female sexual dysfunction: Classification, physiology, diagnosis and treatment
}

\author{
Rebecca H Gladu MD
}

RH Gladu. Female sexual dysfunction: Classification, physiology, diagnosis and treatment. J Sex Reprod Med 2002;2(1):21-27.

Female sexual dysfunction is a common disorder and is classified into four major categories. To successfully diagnose and treat these disorders, physicians must have a working knowledge of the anatomy and physiology of the female sexual response. In addition, the physician must identify the cause of the dysfunction and tailor the treatment to the specific cause. The present review defines and classifies female sexual dysfunction, discusses the pertinent anatomical and physiological features, identifies the four causes of female sexual dysfunction and presents a diagnostic approach for the practicing physician.

\section{Classification, physiologie, diagnostic et traite-} ment de la dysfonction sexuelle chez la femme

RÉSUMÉ : La dysfonction sexuelle chez la femme est un trouble courant classé dans quatre catégories principales. Pour bien diagnostiquer et traiter ce trouble, le médecin doit posséder une connaissance pratique de l'anatomie et de la physiologie de la réaction sexuelle féminine. De plus, il doit repérer la cause de la dysfonction et adapter le traitement en conséquence. La présente analyse définit et classe la dysfonction sexuelle chez la femme, traite des fonctions anatomiques et physiologiques pertinentes, précise les quatre causes de la dysfonction sexuelle chez la femme et présente une démarche diagnostique pour le médecin en exercice.

Key Words: Female sexual dysfunction; Quality of life

K nowledge about female sexual dysfunction is important for the practicing physician. Patients with sexual dysfunction suffer from a compromised quality of life. Physicians caring for women should know how to diagnose and treat this problem. In addition, physicians should screen for female sexual dysfunction, which is most effectively done during a well-woman examination.
The present article fully reviews the subject of female sexual dysfunction for the practicing physician. The goals of the present review are to define and classify female sexual dysfunction, discuss the prevalence of the disorder, review the anatomical and the physiological aspects of the female sexual response, identify four causes of sexual dysfunction, offer a diagnostic approach and discuss the problem-specific treatment options available today.

Family Practice Residency Program, Department of Family Practice and Community Medicine, University of Texas, Houston, USA

Correspondence: Dr Rebecca H Gladu, 6431 Fannin Street, Jesse Jones Library, Suite 308-S, Houston, Texas 77030, USA.

Telephone 713-500-7610, fax 713-500-7619, e-mail Rebecca.H.Gladu@uth.tmc.edu 
TABLE 1 Classification of female sexual dysfunction:
1999 Consensus Classification System

Sexual desire disorders

Hypoactive sexual desire disorder

Sexual aversion disorder

Sexual arousal disorder

Orgasmic disorder

Sexual pain disorder

Dyspareunia

Vaginismus

Other sexual pain disorders

\section{DEFINITION OF FEMALE} SEXUAL DYSFUNCTION

Female sexual dysfunction was defined and classified by the Consensus Development Panel on Female Sexual Dysfunction in 1998 (Table 1) (1). During this meeting, experts in the field of sexual dysfunction, including urologists, psychologists and psychiatrists, developed a definitive classification system. This new system replaced the previous two confounding definitions in the International Classification of Diseases, ninth revision, and Diagnostic and Statistical Manual of Mental Disorders, fouth edition (2), systems. According to the new classification, there are four distinct female sexual disorders: sexual desire disorder, sexual arousal disorder, orgasmic disorder and sexual pain disorder.

- Sexual desire disorders

- Hypoactive sexual desire disorder is the persistent or recurrent deficiency (or absence) of sexual fantasies or thoughts, and/or desire for or receptivity to sexual activity, which causes personal distress.

- Sexual aversion disorder is the persistent or recurrent phobic aversion to and avoidance of sexual contact with a sexual partner, which causes personal distress.

- Sexual arousal disorder

Sexual arousal disorder is the persistent or recurrent inability to attain or maintain sufficient sexual excitement, causing personal distress. This distress may be expressed as a lack of subjective excitement, or genital (lubrication or swelling) or other somatic responses.

\section{- Orgasmic disorder}

Orgasmic disorder is the persistent or recurrent difficulty, delay in or absence of attaining orgasm following sufficient sexual stimulation and arousal, which causes personal distress.

\section{- Sexual pain disorder}

Sexual pain disorder is classified into three subcategories: dyspareunia, vaginismus and noncoital sexual pain disorder.

- Dyspareunia is the recurrent or persistent genital pain associated with sexual intercourse.
- Vaginismus is the recurrent or persistent involuntary spasm of the musculature of the outer third of the vagina that interferes with vaginal penetration, which causes personal distress.

- Noncoital sexual pain disorder is the recurrent or persistent genital pain induced by noncoital sexual stimulation.

Interestingly, male sexual dysfunction was formally classified in 1992, only six years earlier than female sexual dysfunction.

\section{PREVALENCE}

Female sexual dysfunction is age-related, progressive and highly prevalent, affecting $30 \%$ to $50 \%$ of women $(1,3)$. The overall prevalence of sexual dysfunction in American women is $43 \%$. One of three women who report some sexual dysfunction lack sexual interest, $25 \%$ do not experience orgasm, 20\% report lubrication difficulties and $20 \%$ find sex not to be pleasurable. Despite this overwhelming prevalence, only one of 10 women seeks help for the problem. The Analysis of National Health and Social Life Survey (4) concluded that sexual dysfunction is an important public health concern for both sexes. In a survey of 1749 women and 1410 men aged 18 to 59 years, it was found that the prevalence of sexual dysfunction is $43 \%$ in women and $31 \%$ in men (4).

Lubrication or arousal difficulties are prominent in this disorder. In women of reproductive age, $14 \%$ report a persistent lack of lubrication and $23 \%$ have intermittent lubrication difficulties. However, in postmenopausal women, the problem is much more prominent, with $44.2 \%$ having persistent lubrication problems.

The incidence of female sexual dysfunction increases with age and after menopause. One of three patients lacks sexual desire, one of four has problems with orgasm and one of six finds sex to be painful. In women older than 60 years of age, $62 \%$ to $85 \%$ complain of sexual dysfunction of any type. These numbers mean that over 30 million American women report sexual dysfunction (5).

Important in the understanding of the prevalence of sexual dysfunction is the apparent under-reporting of this problem to the patient's physician. In a study of antidepressant-induced sexual dysfunction (6), researchers concluded that sexual dysfunction is often underestimated. In their survey, Montejo-Gonzalez et al (6) found the rate of women spontaneously reporting their own sexual problems to a physician to be only $14.2 \%$. Conversely, $58.14 \%$ reported their sexual dysfunction via questionnaire (6). Perhaps patients are not comfortable discussing this matter with their physicians, and physicians are uncomfortable discussing these issues with their patients (particularly male physicians).

\section{ANATOMY AND PHYSIOLOGY OF THE FEMALE SEXUAL RESPONSE}

To fully understand the etiology and to tailor the treatment of female sexual disorders, one must understand the anatomy and physiology of the normal female sexual response. 


\section{Anatomy}

Women have two corporal bodies that lie on each side of the clitoris. During sexual arousal, these bodies become engorged (erectile tissue); the mechanism is the equivalent of the penile erection in men.

The structure of the clitoris is very much like that of the penis. Both are made of erectile tissue, but there is more to the clitoris than meets the eye. Research based on autopsies of young women is challenging physicians' earlier understanding of this organ. The clitoris wraps around the outside of the vagina up to $8.75 \mathrm{~cm}$ in a pyramid of tissue whose sole function is to transmit sexual pleasure.

The clitoris is located posterior to the pubic symphysis at the anterior end of the pudendal rim. It consists of a body, two crura and a glans. The body is not free, like that of the penis, but is embedded in the connective tissue of the vulva. The body of the organ is formed by the fusion of the two corpora cavernosa, which differ from those of the penis only in size. It extends from the pubic arch anteriorly and posteriorly, is curved and is about 2 to $2.5 \mathrm{~cm}$ in length. Unlike the penis, the third cavernous body is missing from the clitoris. At the inferior border of the pubis, the two corpora cavernosa separate, bend sharply caudally, laterally and dorsally, and follow the inferior borders of the inferior rami of the pubic bones. These are the crura (legs), and they are covered by ischiocavernosus muscles. A glans is attached to the distal end of the fused corpora cavernosa.

\section{Physiology of the female sexual response}

Lubrication: When the vagina responds to sexual stimulation, its smooth muscles relax, dilating the vaginal walls, increasing blood flow and increasing the production of mucous by the vaginal tissues.

In addition, the cervix secretes cervical mucous. When lubrication or arousal fails due to problems in smooth muscle relaxation or decreased blood flow to the genital region, women can experience sexual arousal disorder.

Hormonal physiology of the sexual response: The female sexual response is regulated by estrogen and testosterone. The ovaries and the adrenal glands are responsible for the secretion of androgen in women. The conversion of estrogens to androgenic hormones also takes place in the liver and peripheral tissue. Androstenedione is produced 50\% in the ovaries and $50 \%$ in the adrenal cortex. Testosterone is produced $25 \%$ in the ovaries, $25 \%$ in the adrenal glands and the remaining $50 \%$ from the conversion of androstenedione in the skin and liver. Testosterone in the circulation is bound to sex hormone-binding globulin (SHBG), and only $1 \%$ to $3 \%$ is present as free testosterone. This free testosterone is the active form that can be measured in the blood. The adrenal gland produces the rest of the androgen hormonal component. At menopause, with senescence of the ovaries, a woman's dehydroepiandrosterone, testosterone and estrogen levels decrease (7).

Testosterone's role in the female sexual response is in the initiation and maintenance of libido and spontaneous arousal (8). It is responsible, in part, for sexual desire, sexu- al fantasy, the capacity for orgasm, sexual energy, the sense of well-being and muscle tone. In addition, the lack of testosterone causes thinning and loss of pubic hair.

Estrogen's role is in spontaneous arousal and in facilitating vaginal lubrication. Adequate estrogen levels are necessary for vaginal lubrication and for avoiding atrophic vaginitis, which may cause insertional dyspareunia. Women with estradiol levels below $50 \mathrm{pg} / \mathrm{mL}$ reported more vaginal dryness, dyspareunia, pain and burning, and lower frequency of coital activity than those with higher estradiol levels. These women with low estrogen reported no problems with libido, response or satisfaction with their sexual lives (9). Estrogen alone is unlikely to improve sexual desire and other sexual problems associated with menopause. A combination of estrogen and androgen therapy may be the best approach to alleviate sexual dysfunction for the postmenopausal woman.

\section{THE FOUR CAUSES OF FEMALE SEXUAL DYSFUNCTION}

Female sexual dysfunction is caused by medical illnesses, psychological illnesses, hormonal deficiencies or the effects of medications.

\section{Medical causes}

A decrease in sexual functioning may be due to medical illnesses. Thyroid dysfunction or pituitary adenomas may cause increased prolactin levels, which decrease sexual desire (10). Hypertension, diabetes, high cholesterol levels, heart disease and prior pelvic surgery can cause genital erectile tissue dysfunction similar to that in men. Patients who have had a myocardial infarction may decrease their sexual activity because they feel that the activity will damage their heart. Neurological demyelinating conditions such as multiple sclerosis can cause sexual dysfunction. In addition, alcohol abuse, or cocaine or other narcotic abuse can reduce libido.

\section{Psychological causes}

Psychological problems are frequently the cause of sexual dysfunction in women. Women with underlying major depression, bipolar disorder, schizophrenia, anxiety or eating disorders may present with sexual difficulties. Stress and interpersonal conflict also contribute to this disorder. Physicians should consider medical causes of dysfunction, but should not ignore these common syndromes. A very thorough social history is the key to uncovering an underlying treatable psychiatric illness or psychological dysfunction.

\section{Hormonal causes}

Menopausal changes: Menopausal changes in the genitourinary system include atrophy of the vagina, the vulva, the urethra and the neck of the bladder. Vaginal atrophy and diminished vaginal lubrication interfere mechanically with sexual comfort and pleasure. These changes are caused directly by estrogen deficiency. Women may experience a reduction in pubic hair, loss of fat and subcutaneous tissue 
from the mons pubis, atrophy of the labia majora, and shortening and loss of elasticity of the vaginal barrel. A decrease in the number of vaginal cells may lead to a decrease in vaginal secretions (3).

The Female Androgen Deficiency Syndrome: The Female Androgen Deficiency Syndrome (FADS), first described in 1943 by Salmon and Geist (11), is a hypoandrogenic state in postmenopausal or postoophorectomy patients. It is defined by low circulating testosterone levels (less than $10 \mathrm{ng} / \mathrm{dL})$. Symptoms may include thinning pubic hair, decreased muscle mass and strength, decreased sexual desire, decreased orgasm, a loss of energy, depressed mood or headaches. FADS responds to testosterone replacement therapy (11).

\section{Medication causes}

There is a long list of medications known to affect the sexual responses of both men and women. The major classes of drugs that cause sexual dysfunction are antihypertensives, antipsychotics and antidepressants. The following is a summary of the most common offending medications used in medical practice.

- Serotonin-selective reuptake inhibitor (SSRI) antidepressants:

- serotonin increases prolactin production and lowers libido;

- direct inhibition of nitric oxide synthetase causes less blood flow to erectile tissue; and

- indirectly reduces dopamine levels and decreases libido.

- Phenothiazines increase prolactin and lower libido.

- Antihypertensives (12):

- methyldopa;

- propranolol, timolol;

- clonidine (centrally acting beta-adrenergic blockers);

- reserpine; and

- spironolactone.

- Progesterone increases SHBG and interferes with the bioavailability of testosterone.

- Estrogen increases SHBG and interferes with the bioavailability of testosterone.

- Oral contraceptive pills increase SHBG and interfere with the bioavailability of testosterone.

- Tamoxifen has estrogen-like effects and binds with estrogen receptors.

- Chemotherapy with cytotoxic drugs lowers free testosterone levels.

Antidepressants are a major cause of sexual dysfunction. Recently, this problem has received a large amount of attention from the press and pharmaceutical companies.

In women, the reduction of libido is associated with the following tricyclic antidepressants:

- amitriptyline (Elavil; Merck Frosst, Canada)

- amoxapine (Asendin; Wyeth-Ayerst, Canada)
- clomipramine (Anafranil; Novartis Pharmaceuticals, Canada)

- desipramine (Norpramin; Aventis Pharma, Canada)

- doxepin (Sinequan; Pfizer, Canada)

- nortriptyline

Other antidepressants associated with sexual problems are as follows:

- imipramine (Tofranil; Novartis Pharmaceuticals, Canada) causes delayed orgasm

- triazolopyridine causes anorgasmia - trazodone

- monoamine oxidase inhibitors cause anorgasmia

- SSRIs

- fluoxetine causes delayed orgasm

- paroxetine causes delayed orgasm

- sertraline causes decreased libido

\section{DIAGNOSTIC APPROACH}

The diagnostic approach consists of taking a thorough history, performing a physical examination, a careful review of medications, taking a thorough social history and a few laboratory assays.

\section{History}

The history may be the most difficult part of the examination for physicians who may be uncomfortable discussing sexual issues, but this must be considered a part of the screening process. Merrill et al (13) have identified three main reasons why physicians fail to take a sexual history: embarrassment, failure to recognize that a sexual history is relevant to the presenting complaint, and inadequate training in human sexuality and appropriate sexual history taking (5).

How does one go about taking a sexual history? One very effective method is to include it as part of the review of the genitourinary system. During a well-woman examination, it is customary to ask a series of questions that easily transcend into questions regarding sexuality. The key here is to be very open, with a matter-of-fact approach. One may begin with questions such as "Are you having any problems urinating? Problems with dribbling urine? Any vaginal discharge or dryness?", which lead to questions such as "Any difficulty with intercourse? Any difficulties reaching orgasm? Any other sexual difficulties?". Here, it is important to emphasize that there are many new treatments that can help the patient if she is encountering any sexual difficulties.

Once it is established that the patient has some sort of sexual difficulty, it is important then to establish what type of sexual dysfunction the patient has. Is it problems with libido, arousal or lubrication, orgasmic dysfunction or pain?

Through the careful taking of sexual history, the problem can be determined. Appropriate questions involve all aspects of the patient's sexual encounters that will help to classify the problem and to guide treatment strategies. The sexual interview should include a chronology of the 


\section{COPYRIGHT PULSUS GROUP INC, = DO NOT COPY}

patient's sexual response. The patient should be seated and clothed for the interview. The tone of the questions should be to ascertain which part of the patient's sexual encounter is unsatisfactory to her. Recall that sexual dysfunction is defined as a problem that causes personal distress.

The sexual history: The following questions should be asked during the taking of sexual history.

- Initiation - who initiates? Is this her preference?

- Frequency of coitus - How does she feel about the frequency?

- Is the type and amount of genital stimulation adequate for her?

- Is lubrication adequate and satisfactory for her before intercourse?

- Length of time of intercourse - Is it too long? Does it become painful?

- Ability to achieve orgasm - Difficult? Easy?

- Does she experience any pain? If so, when? Before, during or after intercourse (5)?

Most patients desiring help in this area are very willing to discuss their sexuality with their physicians. For those who feel uncomfortable with such a discussion, a good substitute for a discussion is the Sabbatsberg Sexual Self Rating Scale (14). This is a simple, acceptable, valid and reliable measure of sexual functioning for premenopausal women (14).

\section{Medication review}

Review with the patient the use of any of the medications known to have an effect on libido, including estrogen, oral contraceptive pills, prolactin, antidepressants, psychoactive medications or tamoxifen.

How does sexual dysfunction present in patients taking antidepressants? Often, patients silently accept sexual dysfunction as a negative side effect of antidepressant therapy, or they discontinue treatment due to side effects and live with their depression.

\section{Social history}

Review any narcotic drug and/or alcohol use. Substance abuse can cause significant decreases in libido and sexual functioning. Next, discuss the patient's assessment of the quality of the relationship with her partner. Is she satisfied with her relationship? Finally, do not forget to explore any previous negative sexual experiences that may be playing a role in the patient's dysfunction. Previous sexual abuse, incest or negative sexual experiences could be at the root of her difficulties.

\section{Physical examination}

During the physical examination, search for clues to etiologies. Does the patient have vaginal atrophy, muscle atrophy or thinning pubic hair? These are signs of FADS. Check for the presence of galactorrhea, a sign of hyperprolactinemia. Note the patient's affect and mental status for a possible psychiatric etiology, such as depression or bipolar disorder. Examine the patient for pelvic pain on the bimanual exam- ination. Has the patient had previous breast cancer or mastectomy, and is she taking tamoxifen? Check for any surgical scars. Has she had previous gynecological or urological surgery?

\section{Laboratory assays}

Few laboratory tests are helpful in the diagnosis. Part of the routine evaluation includes measuring the levels of thyroidstimulating hormone and prolactin. If there are clinical signs of FADS, an analysis of the level of free testosterone is indicated. The average cost of a free testosterone level analysis is approximately US\$65.00. The normal range is 20 to $50 \mathrm{ng} / \mathrm{dL}$. Concentrations vary, with morning levels being the highest. A morning level of less than $10 \mathrm{ng} / \mathrm{dL}$ is diagnostic of FADS. Premenopausal women may have a normal diurnal variation between 13 and $52 \mathrm{ng} / \mathrm{mL}$ (15).

Additional testing: Tests such as genital blood peak systolic velocity (duplex ultrasonography study), vaginal $\mathrm{pH}$, intravaginal pressure-volume changes (measures vaginal compliance) and genital vibratory perception thresholds are investigative methods used to quantify aspects of the female sexual response. In addition to quantifying the degree and the nature of dysfunction, these tests may be useful in evaluating the efficacy of newer pharmacotherapies. At this time, these measurements are not available for the practicing physician (3).

\section{TREATMENT STRATEGIES}

Physicians should tailor the treatment of female sexual dysfunction to the underlying cause.

\section{Medical therapy}

The correction of medical disorders, such as hyperprolactinemia, is essential. Just as with erectile dysfunction, elimination of risk factors, such as tobacco use and obesity, and attention to exercise and stress management are an important part of treatment in this patient population.

\section{Medication change}

If there is a clear causal relationship, consider discontinuing or changing the suspected medication. Many antidepressants will interfere with orgasmic function. Newer antidepressants, such as mirtazapine and bupropion $(16,17)$ may not cause sexual dysfunction. In general, all SSRIs, even those with dual action, can be implicated. A good strategy is to suggest changing to another SSRI after a break unless this change is contraindicated. No SSRIs are without side effects. Just like fluoxetine, mirtazapine affects the serotonin system in the brain (18).

In a recent study (19), 66 nondepressed men and women with nonphysiological orgasmic delay or inhibition, aged 23 to 65 years, were given a placebo for four weeks and bupropion hydrochloride sustained-release tablets for eight weeks. One of three female subjects responded with overall sexual satisfaction and satisfaction with the intensity of orgasm. Seventy per cent of subjects reported improvement in libido, arousal or orgasmic function during bupropion 


\section{COPYRIGHT PULSUS GROUP INC, = DO NOT COPY}

administration. Response was seen as early as two weeks after the beginning of treatment. By the end of the eightweek treatment phase, the response rate indicated a more than two-fold increase in the frequency of interest in sexual activity, almost double the frequency of sexual arousal and more than twice the number of sexual fantasies (19).

Secondly, be sure that the antidepressant medication is truly the cause of the sexual dysfunction. Rule out a hormonal problem, then try another antidepressant.

Should a particular antidepressant medication be identified as the cause of the sexual dysfunction in the patient, switching the antidepressant medication to an agent with fewer sexual side effects is an option. Algorithms are available for changing from any antidepressant to one such as bupropion or mirtazapine.

\section{Psychosexual therapy}

When organic causes of sexual dysfunction have been ruled out and a psychological diagnosis has been made, the physician must tailor the therapy. Treat depression, if present, with psychotherapy and medication; however, many antidepressants may cause sexual dysfunction. This treatment requires careful and frequent monitoring of the patient, paying close attention to drug side effects and depression improvement. It requires a supportive physician and a good doctor-patient relationship.

Relationship discord may be the only cause of sexual dysfunction in some patients. Often, sexual problems are symptoms of a relationship under stress. Referral to individual or couples' therapy can be beneficial.

If the problem identified is stress, consider stress reduction, biofeedback, counselling or psychotherapy. Establish a good referral relationship with a therapist who is comfortable treating women with sexual dysfunction.

Frequently, young women feel discomfort with their bodies and are uncomfortable expressing sexual needs to their partner. These patients may benefit from personalized instruction from a sex therapist or from couples' therapy.

Keep in mind that patients may need a combination of medical therapy and psychotherapy.

\section{Vasoactive drugs}

Vasoactive drugs may prove to be efficacious for women with sexual arousal dysfunction; however, there are no approved vasoactive drugs for women. Sildenafil may work in a similar fashion in women as that in men by increasing blood flow to the genital area. This increase in blood flow may in turn increase lubrication, sensation and engorgement of the genitalia, and improve the potential for orgasm. Anecdotal evidence suggests that it is efficacious in treating antidepressant-induced sexual dysfunction.

\section{Endocrine therapy}

Endocrine therapy is helpful for postmenopausal women and for women with FADS.

Postmenopause: When postmenopausal sexual difficulty is caused by vaginal atrophy, decreased lubrication or urogenital atrophy, hormonal replacement therapy is indicated.
Postmenopausal or postoophorectomy patients benefit from appropriate hormone replacement therapy. Additional vaginal estrogen therapy may be needed in the case of vaginal atrophy.

\section{FADS}

FADS responds to testosterone replacement therapy in physiological doses. An estrogen-androgen hormone replacement therapy can also be used because estrogen and testosterone act synergistically to treat this syndrome. Testosterone cream or gel can be dispensed from a compounding pharmacy at the recommended dose of testosterone for women of 0.25 to $0.50 \mathrm{mg} / \mathrm{day}$ and be topically applied to the vulva or inner thigh.

No discussion of androgen replacement in women would be complete without the mention of the physiological consequences of this treatment. Unfortunately, these drugs have been studied in superphysiological doses, such as methyltestosterone $5 \mathrm{mg} /$ day orally. In addition, many of these medications have been studied only in the oral route in which the first-pass effect on the liver must be considered. Furthermore, methyltestosterone should be avoided because it is hepatotoxic. In Canada, testosterone undecanoate (Andriol; Organon, Canada) is used. It is absorbed by the lymphatic system and avoids the liver. It is safe and inexpensive at a dose of $40 \mathrm{mg} /$ day given on alternate days. Benefits of androgen therapy: The benefits of androgen therapy are as follows:

- increased bone mass;

- reduction of urinary calcium excretion and bone resorption; and

- decreased urogenital aging.

Negative side effects of androgen therapy: The negative side effects of androgen therapy are as follows (20-22):

- poor lipoprotein effects - low density lipoprotein, triglyceride, total cholesterol and apolipoprotein-B levels increase; high density lipoprotein level decreases;

- may cause glucose intolerance;

- uncertain effect on breast tissue; and

- virilizing effects - acne, hirsutism, deepening of the voice and clitoromegaly at superphysiological doses.

The available oral androgens in the United States are available in doses that far exceed the physiological needs of women (0.5 to $0.8 \mathrm{mg} /$ day) (11). In addition, testosterone injections are also available; however, they are available only in doses appropriate for men.

Fortunately, for women with FADS, testosterone is available from compounding pharmacies as a testosterone cream or gel. The dose range available is the recommended physiological replacement dose of 0.25 to $0.8 \mathrm{mg} /$ day with no virilizing side effects. The therapeutic dose at which beneficial effects on female libido can be achieved without incurring unwelcome masculinizing side effects is usually attained at one-tenth of the dose that is generally administered to hypogonadal men (23). 
Testosterone cream or gel: The testosterone cream or gel can be formulated as follows:

- methyltestosterone $0.25 \mathrm{mg} / 0.1 \mathrm{~mL}$ in pluronic lecithin organogel; and

- testosterone propionate $0.25 \%$ to $0.5 \%$ emollient cream.

Directions for use: Apply to the vulva daily for two weeks, then daily or every other day to the inner thigh for 30 days and continue use as needed. It is important that the cream be kept refrigerated and stirred before use (5). A transdermal formulation of testosterone at $300 \mu \mathrm{g} /$ day will be available soon as an alternative method of delivery (24).

Caution: The administration of low nonvirilizing doses of testosterone to healthy women with demonstrably low lev-

\section{REFERENCES}

1. Berman LA, Berman JR, Chhabra S, Goldstein I. Novel approaches to female sexual dysfunction. Expert Opin Investig Drugs 2001;10:85-95.

2. Diagnostic and Statistical Manual of Mental Disorders, 4th edn. Washington: American Psychiatric Association, 1994.

3. Berman JR, Berman LA, Wervin TJ, Flaherty EE, Leahy NM, Goldstein I. Clinical evaluation of female sexual function: effects of age and estrogen status on subjective and physiologic sexual responses. Psychosom Med 1983;45:259-69.

4. Laumann EO, Paik A, Rosen RC. Sexual dysfunction in the United States: prevalence and predictors. JAMA 1999;281:1174.

5. Rako S. The Hormone of Desire. New York: Random House, 1996.

6. Montejo-Gonzalez AL, Llorca G, Izquierdo JA, et al. SSRIinduced sexual dysfunction: fluoxetine, paroxetine, sertraline, and fluvoxamine in a prospective, multicenter, and descriptive clinical study of 344 patients. J Sex Marital Ther 1997;23:176-94

7. Speroff L, Glass R, Kase N. Clinical Gynecologic Endocrinology and Infertility. Amsterdam: Wolters Kluwer, 1999.

8. Seidman SN. Hormonal aspects of sexual dysfunction: the therapeutic use of exogenous androgens in men and women. Curr Psychiatry Rep 2000;2:215-22.

9. Sarrel PM. Effects of hormone replacement therapy on sexual psychophysiology and behavior in postmenopause. J Womens Health Gend Based Med 2000;9(Suppl 1):S25-32.

10. Weizman R, Weizman A, Levi J, et al. Sexual dysfunction associated with hyperprolactinemia in males and females undergoing hemodialysis. J Urol 2000;163:888-93.

11. Salmon UJ, Geist SH. Effect of androgens upon libido in women. 26th Annual Meeting of the Association for the Study of Internal Secretions. Atlantic City, June 9, 1942.

12. Phillips NA. Female sexual dysfunction: Evaluation and treatment. Am Fam Physician 2000;62:127-35. els of testosterone is considered to be safe. However, testosterone administration poses definite risks for women with congestive heart failure and some cancers. Hormones should not be prescribed for any patient unless she has a demonstrable deficiency (23).

Many authors have discussed the idea of including testosterone therapy as part of routine hormone replacement therapy for postmenopausal women $(5,22)$. This hormone replacement therapy would involve the use of estrogen, progesterone and low dose testosterone cream.

ACKNOWLEDGEMENTS: The author acknowledges Dr Linda Nieman for her help and support in the preparation of this manuscript.

13. Merrill JM, Laux LF, Thornby JI. Why doctors have difficulty with sex histories. South Med J 1990;83:613-7.

14. Garratt AM, Torgerson DJ, Wyness J, Hall MH, Reid MD. Measuring sexual functioning in premenopausal women. Br J Obstet Gynaecol 1995;102:311-6.

15. Zumoff B, Strain GW, Miller LK, Roser W. 24 hour mean plasma testosterone concentration declines with age in normal premenopausal women. J Clin Endocrinol Metab 1995;80:1429-30.

16. Croft H, Settle E Jr, Houser T, Batey SR, Donahue RM, Ascher JA. A placebo-controlled comparison of the antidepressant efficacy and effects on sexual functioning of sustained-release bupropion and sertraline. Clin Ther 1999;21:643-58.

17. Gelenberg AJ, Laukes C, McGahuey C, et al. Mirtazapine substitution in SSRI-induced sexual dysfuntion. J Clin Psychiatry 2000;61:356-60.

18. Anttila SA, Leinonen EV. A review of the pharmacological and clinical profile of mirtazapine. CNS Drug Rev 2001;7:249-64.

19. Modell JG, May RS, Katholi CR. Effect of bupropion-SR on orgasmic dysfunction in nondepressed subjects: a pilot study. J Sex Marital Ther 2000;26:231-40.

20. Sands R, Studd J. Exogenous androgens in postmenopausal women. Am J Med 1995;98:76S-9S.

21. Davis SR. The clinical use of androgens in female sexual disorders. J Sex Marital Ther 1998;24:153-63.

22. Sarrell PM. Cardiovascular aspects of androgens in women. Semin Reprod Endocrinol 1998;16:121-8.

23. Singer HS, Owett T. The Female Androgen Deficiency Syndrome. J Sex Marital Ther 1993;19:3-24.

24. Shirfen JL, Braunstein GD, Simon JA, et al. Transdermal testosterone treatment in women with impaired sexual function after oophorectomy. N Engl J Med 2000;343:682-8.

\section{ADVERTISER'S INDEX}

The Journal of Sexual \& Reproductive Medicine is a peer review journal, financed almost entirely through advertising. The companies advertising in this journal have recognized the need to support high quality, Canadian, ethical journals and to promote publishing of Canadian medical research. Pulsus Group Inc and the readers of the Journal of Sexual \& Reproductive Medicine appreciate the support of the following companies for making this issue possible:

Janssen-Ortho

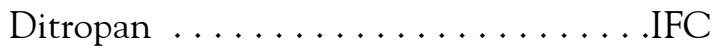

Prescribing information ...........16
Pfizer

Viagra ................... OBC

Prescribing information $\ldots \ldots \ldots . \ldots 39$

\section{Organon}

Marvelon ..................4 